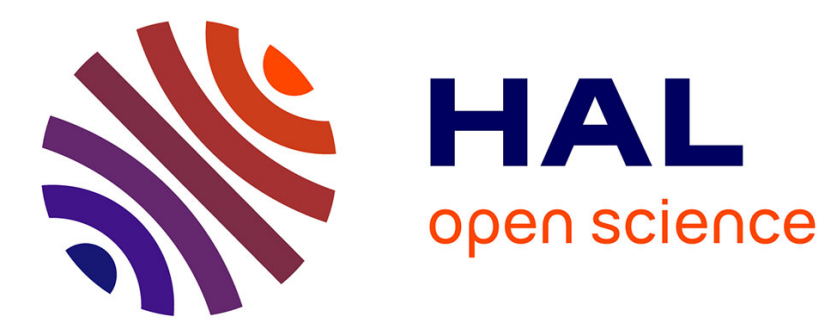

\title{
Electronic properties of epitaxial graphene
}

Claire Berger, Jean-Yves Veuillen, Laurence Magaud, Pierre Mallet, Valerio Olevano, Xavier Blase, Milan Orlita, Paulina Plochocka, Clement Faugeras, Gines Martinez Garcia, et al.

\section{- To cite this version:}

Claire Berger, Jean-Yves Veuillen, Laurence Magaud, Pierre Mallet, Valerio Olevano, et al.. Electronic properties of epitaxial graphene. International Journal of Nanotechnology, 2010, 7 (4-8), pp.383-402. 10.1504/IJNT.2010.031726 . hal-01002862

\section{HAL Id: hal-01002862 https://hal.science/hal-01002862}

Submitted on 1 Dec 2016

HAL is a multi-disciplinary open access archive for the deposit and dissemination of scientific research documents, whether they are published or not. The documents may come from teaching and research institutions in France or abroad, or from public or private research centers.
L'archive ouverte pluridisciplinaire HAL, est destinée au dépôt et à la diffusion de documents scientifiques de niveau recherche, publiés ou non, émanant des établissements d'enseignement et de recherche français ou étrangers, des laboratoires publics ou privés. 


\section{Electronic properties of epitaxial graphene}

Claire Berger, Jean-Yves Veuillen, Laurence Magaud, Pierre Mallet, Valerio Olevano, Xavier Blase, M. Orlita, P.Plochocka, C. Faugeras, G. Martinez, Marek Potemski, Cécile Naud, Laurent Lévy and Didier Mayou

Institut Néel CNRS and UJF

LCNMI

25 Avenue des Martyrs 38042 Grenoble Cedex 9

*Corresponding author: didier.mayou@grenoble.cnrs.fr

Abstract: It has been known for almost 25 years that high temperature treatment of polar faces of $\mathrm{SiC}$ crystals lead to the graphitization of the surface as a consequence of Si preferential sublimation. The group of Walt de Heer in Atlanta has proposed to use this procedure to obtain macroscopic multilayer graphene samples. This has lead to a renewal of interest for the study of the graphitic layers grown on the SiC surfaces. Due to the polar nature of the material, the $6 \mathrm{H}(4 \mathrm{H})-\mathrm{SiC}$ substrate present two non-equivalent surfaces: the (0001) is known as the Si face, and the (000-1) one is the $C$ face. It turns out that the structure and the properties of the multilayer graphene samples obtained on those two surfaces are rather different. In particular the multilayer graphene samples grown on the C-terminated face presents electronic properties that are rather similar to those of monolayer graphene with very high mobility. This is shown by transport and optical experiments under high magnetic field. This surprising result is explained by the rotational stacking sequence, observed experimentally. which leads to an electronic decoupling as demonstrated by ab-initio band structure calculations. 


\section{Introduction}

It has been known for almost 25 years that high temperature treatment of polar faces of $\mathrm{SiC}$ crystals lead to the graphitization of the surface [1] as a consequence of $\mathrm{Si}$ preferential sublimation. Later works [2] have shown that the number of C planes could be controlled by the annealing temperature, and that the electronic structure of multilayer samples was similar to that of graphite. The group of Walt de Heer in Atlanta has proposed to use this procedure to obtain macroscopic multilayer graphene samples [3]. This has lead to a renewal of interest for the study of the graphitic layers grown on the $\mathrm{SiC}$ surfaces. The material obtained in this way is known as Few Layers Graphene (FLG). Commercial wafers of the hexagonal polytypes $6 \mathrm{H}$ and $4 \mathrm{H}-\mathrm{SiC}$ are commonly used as substrate, and the graphitization is completed by thermal treatment at temperatures in excess of $1200^{\circ} \mathrm{C}$. The number of C planes depends not only of external parameters such as temperature and pressure, but also of the initial substrate surface (for a review see [4]). Due to the polar nature of the material, the $6 \mathrm{H}(4 \mathrm{H})-\mathrm{SiC}$ substrate present two non-equivalent surfaces: the (0001) is known as the Si face, and the (000-1) one is the $\mathrm{C}$ face. It turns out that the structure and the properties of the FLG obtained on those two surfaces are different, as discussed below. This sublimation technique is appealing since it can lead in principle to the formation of large area graphene samples convenient for applications [5]. However, the materials obtained in this way are not isolated graphene planes and the question of their interaction with their environment needs to be investigated in order to reveal any possible perturbation to the ideal case.

In section II, we consider the interaction with the $\mathrm{SiC}$ substrate, from which the FLG's are grown. This point is addressed for the case of FLG grown on the Si face, where it will be shown that there is a strong coupling between the first $\mathrm{C}$ plane and the substrate, but that the characteristic features of graphene are recovered from the second plane. In section III we consider the interaction with adjacent $\mathrm{C}$ planes. This is an important point since i) typical macroscopic samples are, at least locally, several layer thick and ii) it is known that the electronic properties of graphite-like (AB) stacked multilayer graphene are markedly different from those of single layer ones. We shall show in section III that due to the peculiar growth process of FLG on the substrate C face there is an effective electronic decoupling of adjacent $\mathrm{C}$ planes that helps restoring single layer behaviour even in thick samples. Finally in section IV we present with more details the transport properties of epitaxial graphene on the $\mathrm{C}$-face for which remarkable transport characteristics are demonstrated. 


\section{Graphene-substrate interaction: FLG's on the $4 \mathrm{H}(6 \mathrm{H})-\mathrm{SiC}(0001)$ substrate.}

Graphitization of the substrate Si face starts from the so-called $6 \sqrt{3} \times 6 \sqrt{3} R\left(30^{\circ}\right)-6 R 3$ in short- $\mathrm{SiC}$ surface reconstruction. It has long been recognized $[1,2,6]$ that this reconstruction was induced by a graphitic C-plane lying on top of the substrate. The Csurface plane is rotated by $30^{\circ}$ with respect to the underlying substrate which gives rise to this large common unit cell : a 6R3 with respect to the SiC substrate and a (13x13) with respect to the graphitic plane. However, apart from these geometrical considerations, little was known either on the actual interface structure or on the strength of the coupling between the graphitic layer and the substrate. In section II-A, we present DFT calculations on model systems which explain important features of recent experimental data. STM results supporting the same findings are presented in section II-B.

\section{1: Atomic and electronic structure studies from ab initio calculations}

Interaction of one and two $\mathrm{C}$ layers with the $\mathrm{Si}$ face of the $\mathrm{SiC}$ substrate has been investigated in the actual $6 \mathrm{R} 3$ cell. The ab initio calculations have been performed using the code VASP [7] that enables the determination of the atomic equilibrium positions and then of the associated electronic structure. The interface cell contains more than 1200 atoms. This is very large for ab initio approaches.

Figure 1 : Morphology and electronic structure of one and two $\mathrm{C}$ layers on the $\mathrm{Si}$ face of $\mathrm{SiC}$ (0001). (a) ab initio total charge density just above one $\mathrm{C}$ layer on top of $\mathrm{SiC}$, (b) ab initio total charge density just above the surface plane for two $\mathrm{C}$ layers on top of $\mathrm{SiC}$. The interface $6 \mathrm{R} 3$ cell is indicated in yellow and the cell of the apparent $6 \times 6$ modulation is given in red. (c) cross section of the ab initio total charge density in one $6 \mathrm{R} 3$ unit cell for $2 \mathrm{C}$ layers on top of $\mathrm{SiC}$. It shows the bump associated to grain boundary junctions that are responsible for the $6 \times 6$ apparent modulations. (d) band structure for one $\mathrm{C}$ layer on $\mathrm{SiC}$. (e) band structure for two $\mathrm{C}$ layers on $\mathrm{SiC}$ that demonstrates the doped graphene nature of the second $\mathrm{C}$ layer.
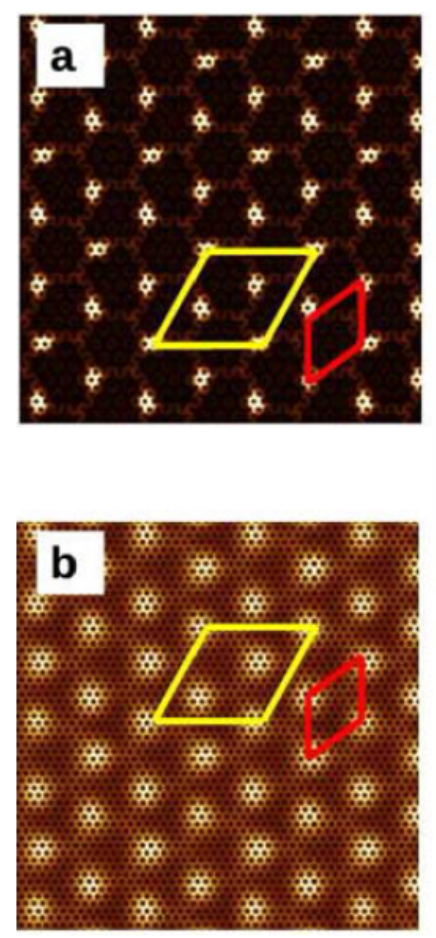
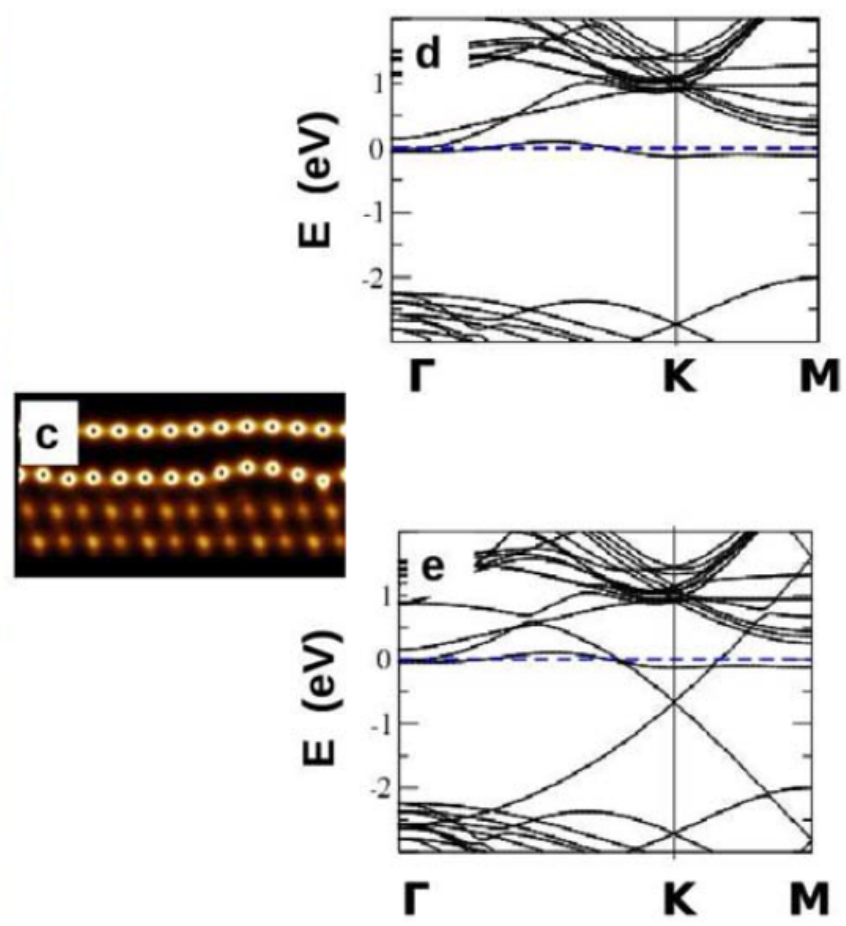
Fig. 1-a shows the total charge density just above one C-plane on top of SiC -its morphology does not change when a second $\mathrm{C}$ plane is added-. It presents a mosaïc structure with grains that correspond to matching areas of the $\mathrm{C}$ honeycomb and the $\mathrm{SiC}$ lattices [8]. In these regions, strong $\mathrm{C}-\mathrm{Si}$ bonds are formed. The local symmetry within the grains corresponds to a $\sqrt{3} \times \sqrt{3} \mathrm{R}\left(30^{\circ}\right)$. It is shifted in adjacent grains and the boundaries accommodate the shifts. No $\mathrm{C}-\mathrm{SiC}$ bonds are formed there, as a consequence, the $\mathrm{C}$ atoms lay higher above the $\mathrm{SiC}$ surface. Boundary junctions create an apparent $6 \times 6$ modulation (red diamond in Fig.1-a) that is also seen on STM images [6]. The calculated corrugation of the $\mathrm{C}$ plane is rather large $-1.5 \AA$ - in agreement with STM data. The atomic structure of the first $\mathrm{C}$ layer on the $\mathrm{Si}$ face of $\mathrm{SiC}$ derives from a honeycomb lattice but it is strongly disturbed by the formation of covalent bonds with the substrate. The corresponding band structure (Fig.1-d) does not show the linear dispersion characteristic of graphene. The dispersion curves presented here have been calculated in the $\sqrt{3} \times \sqrt{3} \mathrm{R}\left(30^{\circ}\right)$ matching geometry and not in the actual 6R3 cell [9]. This is because calculations for more than 1200 atoms are really heavy. A few k points close to the Dirac point have been performed in the complex structure, they confirm the present finding.

As a conclusion, the first $\mathrm{C}$ layer on top of the $\mathrm{Si}$ terminated $\mathrm{SiC}$ face is not a graphene layer. As we will discuss it in the following, it passivates the SiC surface so that graphene properties are recovered from the second $\mathrm{C}$ plane. For these reasons, we called it a buffer layer. The non-graphene behaviour of the buffer layer has also been demonstrated by ARPES measurements that found no evidence of p-states and then no linear dispersion [8-10].

When a second C-plane is added on top of the buffer layer, it smoothly follows its morphology so that soft $6 \times 6$ ripples [8] are induced in the otherwise graphene like honeycomb lattice - see top view in Fig.1-b and cross section in Fig.1-c - . This 6x6 modulation is also obvious on STM images. The calculated corrugation is much smaller $0.5 \AA-$ in the second plane, in agreement with STM. The band structure -in the local $3 \mathrm{x} \sqrt{3} \mathrm{R}\left(30^{\circ}\right)$ matching geometry- shows a linear dispersion characteristic of graphene (Fig. 1-e) [9]. The main conclusion is that the $\mathrm{C}$-second plane indeed has graphene electronic structure but it is doped since the Dirac point (the energy which separates electrons and holes) lies $0.4 \mathrm{eV}$ below the Fermi level. A charge transfer from the interface states to the $\mathrm{C}$ layer is responsible for this doping. Both results -linear dispersion and doping from interface states- are in agreement with ARPES data [8-10].

\section{2 : STM studies of mono and bilayer graphene:}

In this paragraph, we present atomic resolution images of the monolayer and bilayer graphene phases, which according to the previous discussion should correspond to the second and third C-plane above the $\mathrm{SiC}$ substrate. For the buffer layer or 6R3 reconstructed surface, an apparent (6x6) superstructure is usually found [6] but the honeycomb atomic structure of the strongly bound first C-plane is not observed in STM images. Both findings are consistent with the model of section II-A, the latter resulting from the absence of $\mathrm{C}_{\mathrm{z}}$-states in the vicinity of the Fermi level for the buffer layer.

A low bias STM image of single layer graphene is presented in figure 2-a. It shows a small scale atomic structure (with period $\approx 2.44 \AA$ ), superimposed on a large scale structure (period $\approx 1.8 \mathrm{~nm}$ ) which is reminiscent of the apparent (6x6) modulation of the 
underlying buffer layer. The small scale structure has the expected period for the graphene lattice. Moreover it presents a honeycomb contrast. It means that the local density of states (LDOS) of A and B sublattices are essentially equivalent -at least in the vicinity of the Fermi level-, which is a characteristics of monolayer graphene [13]. Figure 2-b displays an image taken at the same location but at slightly higher bias. In addition of the aforementioned features, localized atomic scale structures show up in these images, one of them is indicated by the blue arrow. These structures are due to electronic states which exist in the interface plane below the graphene layer [13,14]. This indicates that there exists a -somewhat disordered- distribution of low-lying interface states in this system. Their influence on the physical properties of the graphene layer remains an open issue.

Figure 2: STM images of FLG grown on the $\mathrm{Si}$ face of $6 \mathrm{H}-\mathrm{SiC}$. (a) monolayer graphene, size $8 \times 4$ $\mathrm{nm}^{2}$, sample bias $-100 \mathrm{mV}$. (b) same as (a) for sample bias $-300 \mathrm{mV}$. Additional structures appear in the image (one is shown by the arrow). (c) image of a boundary between bilayer graphene (B) and monolayer graphene (M). Image size: $10 \times 4 \mathrm{~nm}^{2}$, sample bias: $-200 \mathrm{mV}$. The inset (size: $1.8 \times 1.2 \mathrm{~nm}^{2}$ ) displays the atomic resolution on the $\mathrm{B}$ terrace. The arrows indicate the height difference between the $\mathrm{M}$ and $\mathrm{B}$ terraces.
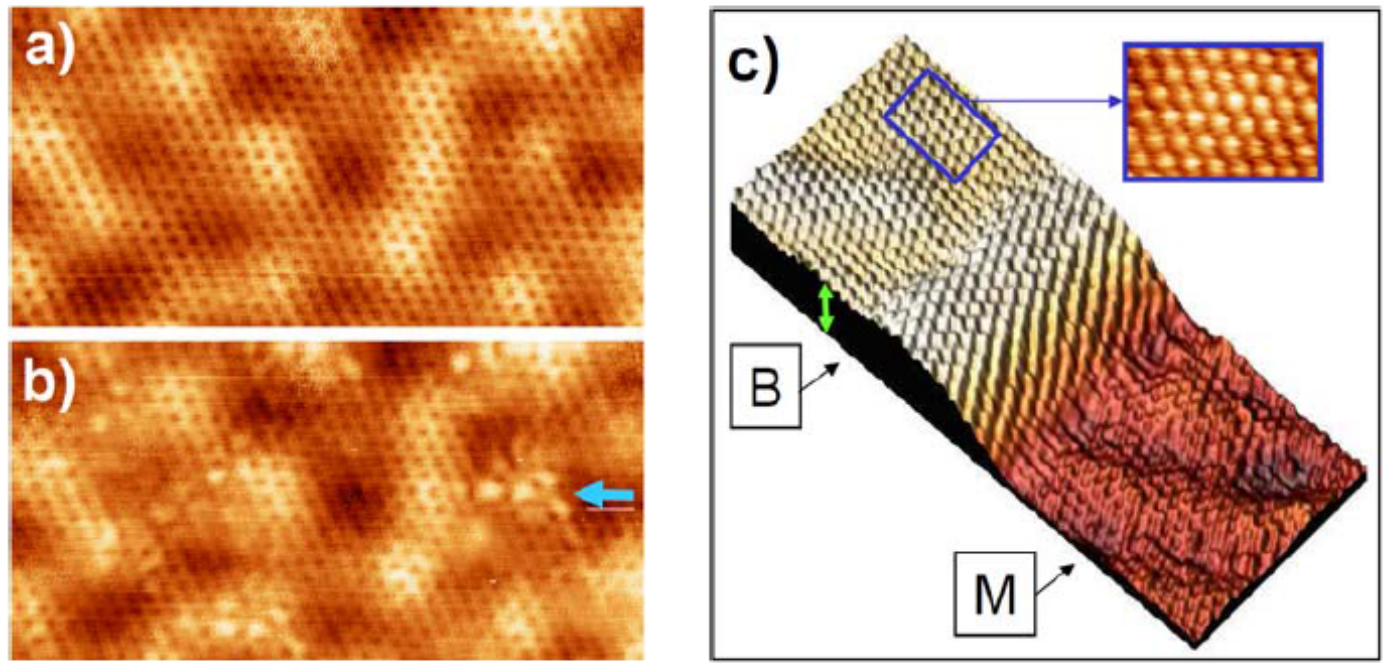

In figure 2-c, we present a low bias image taken at a boundary between a bilayer graphene phase (upper part of the image, denoted as B) and a monolayer graphene phase (lower part of the image, denoted as $\mathrm{M}$ ). The height difference between these two terraces (green arrow) is in the range $3.27 \pm 0.15 \AA$, which is close to the value of the inter-plane distance in graphite $(3.35 \AA)$. The inset is a zoom on the B region. It shows a hexagonal lattice with the period expected for graphene/graphite, but with a simple triangular contrast as usually found on graphite surfaces [15] (the honeycomb contrast is still observed on the lower $\mathrm{M}$ terrace). This demonstrates that the A and B sublattices in the surface plane of the bilayer are not equivalent, as expected for the so-called ' $A B$ stacking' of the carbon planes (the regular one for graphite) [13]. Finally, we notice in this three-dimensional representation that the surface carbon plane is continuous at the boundary. This is a common observation $[3,16]$. 
Low bias STM images can also be used to probe the electronic properties at the Fermi surface (Pedersen). In low bias images of graphene (mono and bilayer) or graphite a socalled $\sqrt{3} x \sqrt{3} R\left(30^{\circ}\right)$ superstructure exists in the vicinity of point defects $[13,17,18,19]$. It derives from the peculiar shape of the Fermi surface of graphene which consists of small pockets located around the $\mathrm{K} / \mathrm{K}$ ' points of the surface Brillouin zone $[18,19,13]$. Alternatively, the observation of these superstructures allows concluding that the Fermi surface of graphene is established for the monolayer and bilayer samples $[19,13]$. Interestingly, this superstructure is not observed close to the boundary in figure 2-c although it is an armchair type edge. On graphite, an atomically sharp armchair edge generates a well developed $\sqrt{3} \times \sqrt{3} \mathrm{R}\left(30^{\circ}\right)$ superstructure [20]. The absence of such feature in figure 2-c therefore suggests that the boundary is a smooth scattering centre. Finally, the Fourier transform analysis of the low bias images reveals fundamental characteristics of the graphene layers on the Si face: doping [19, 21], Fermi velocity [19] and electronic chirality [21].

\section{Interaction between twisted layers formed on the $4 \mathrm{H}(6 \mathrm{H})-\mathrm{SiC}(000-1)$ substrate : electronic decoupling.}

An important conclusion of many experiments -magnetotransport [22, 23], magnetooptical [24] and Raman [25] spectroscopy- performed on FLG's films grown on the $\mathrm{C}$ face of the substrate was that those samples exhibited the characteristic electronic properties of single layer graphene although they were actually several planes thick. Considering that the electronic structure and properties of $\mathrm{AB}$-stacked bilayer graphene are already different from those of monolayer graphene, this result is somewhat surprising. In this section, we show that this effect is related to the specific stacking of the C planes in the FLG's films grown on the C-face. The strict AB-stacking is lost in those samples, and this leads to an electronic decoupling of adjacent layers. Section III-A presents STM data which actually reveal, at the sample surface, a rotation between adjacent layers. DFT calculations illustrating the effect of (large) rotation angle on the electronic structure of a bi(multi)layer are presented in section III-B. Finally, experimental evidence for the electronic decoupling effect due to this rotation is given in section III-C.

\section{1: Twisted surface layers observed by STM:}

An STM image of a FLG sample $(\approx 5 \mathrm{C}$ layers thick) is shown in figure $3-\mathrm{a}$. This is a single terrace, which is divided in domains separated by graphitic structures denoted "beads" and "folds" which are a couple of $\AA$ high (typical height in the 1-10 $\AA$ range in this image). Most of the domains appear as flat, but two of them (indicated as A and B in figure 4) show a hexagonal superstructure with period $2.25 \mathrm{~nm}$ (domain A) or $5.65 \mathrm{~nm}$ (domain B). The superstructures, as well as the beads, are well known features of graphite surfaces (see [26] for a review). For graphite, the superstructures are interpreted as 'Moiré patterns' arising for a rotation of the surface layer -by a couple of degreeswith respect to the underlying 'bulk' planes. We have verified that this interpretation was indeed correct also for our FLG samples. Figure 4-b displays a boundary between a domain presenting a strongly modulated superstructure with period $2.31 \mathrm{~nm}$ (noted as T) and an (almost) flat domain (noted as B). From the period of the superstructure, we can 
compute the expected rotation angle $\theta$ between the surface plane and the sub-surface one in a Moiré model for the $\mathrm{T}$ domain [26]. We find $\theta=6.1^{\circ}$. From the Fourier transform of the image (inset of figure 4), we can estimate the angle between the surface atomic lattices of the $T$ and $B$ domains, which is $6 \pm 1^{\circ}$. This value is consistent with the one obtained by the Moiré model. The validity of this model has been confirmed for superstructures with different periods [27]. Notice that a faint superstructure is also present on the B domain in figure 4-b. Its period is similar to the one for the T domain, but the orientation is different and the modulation amplitude is much smaller (by a factor of 5). This indicates [26] that rotation takes place not only at the surface but also between sub-surface (buried) planes. A distribution of the orientation of the graphene islands with respect to the substrate is indeed observed for the $\mathrm{C}$ face already in the monolayer range [28]. Thus the substrate does not impose the orientation of the graphene layers, at variance with the situation encountered on the Si face.

Figure 3: STM images of FLG grown on the $\mathrm{C}$ face of $6 \mathrm{H}-\mathrm{SiC}$. (a) image for a single terrace, size $120 \times 52 \mathrm{~nm}^{2}$, sample bias : $+500 \mathrm{mV}$. The structures indicated are discussed in the text.

(b) image of a boundary between a modulated domain ( $T$ ) and an (almost) flat region

(B). Image size: $34 \times 19 \mathrm{~nm}^{2}$, sample bias: $-500 \mathrm{mV}$. The inset shows the Fourier transform of the image: two sets of spots (circles) corresponding to the atomic lattice in the $\mathrm{B}$ and $\mathrm{T}$ domains appear, rotated by $6 \pm 1^{\circ}$.
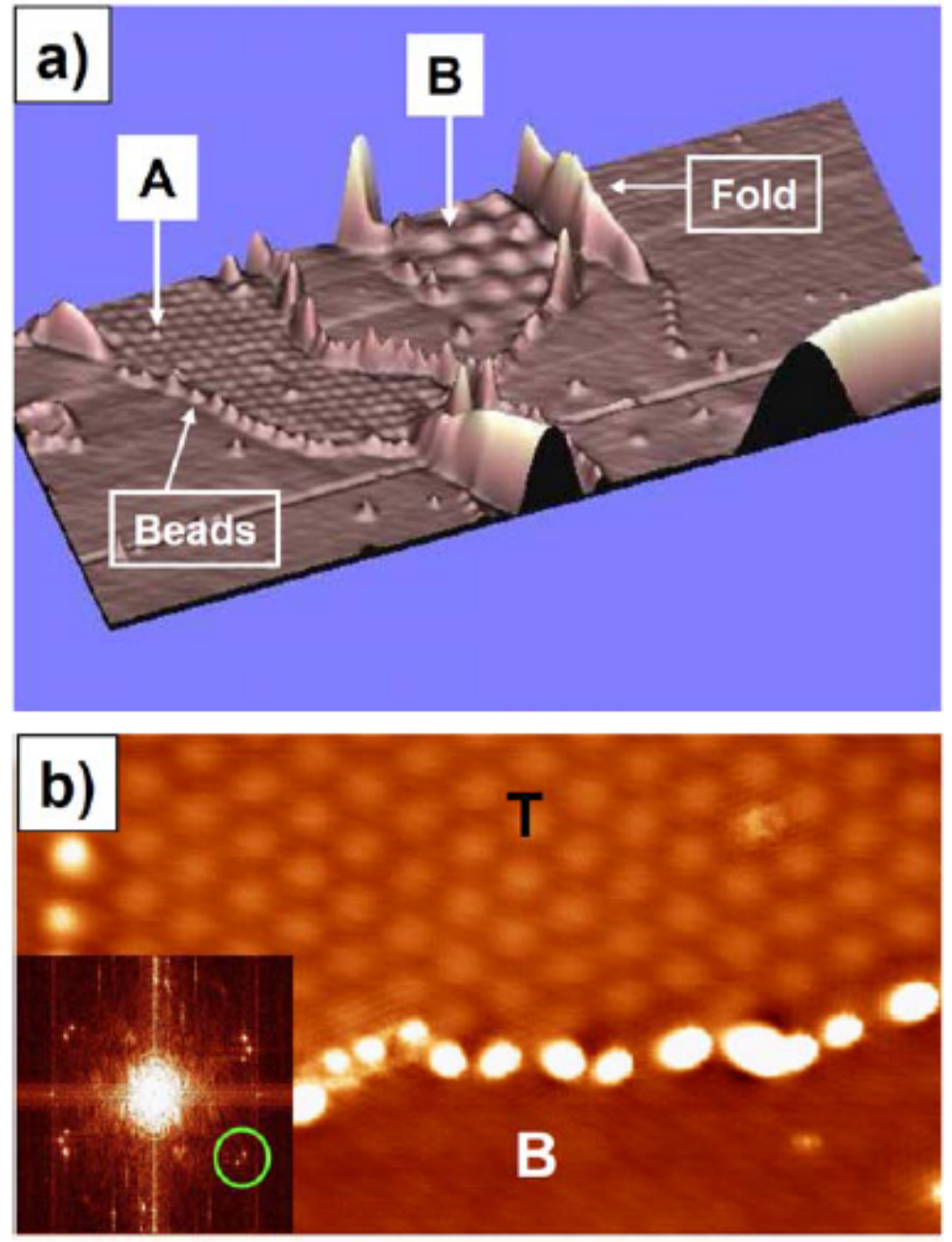
From our measurement [27], we have obtained values of $\theta$ (the rotation angle between adjacent planes) ranging from 1.5 to $19^{\circ}$ in the modulated domains. A rotation between adjacent carbon planes has important consequences for FLG, since it can lead at least close to the Dirac point- to the survival of the characteristic electronic structure of monolayer graphene even in multilayer samples. This will be shown below for large rotation angles. For the relatively small values of $\theta$ (a few degrees) that we have found in our samples, the electronic decoupling effect has been demonstrated analytically $[29,30]$ for bilayers, and it is possibly reflected in atomic resolution images [27].

Finally, notice that the sample of figure 4 has been grown under ultra-high vacuum (UHV) at comparatively low temperature $\left(1150^{\circ} \mathrm{C}\right)$. It turns out that the samples elaborated in secondary vacuum at higher temperature (which will be considered in the following sections) have a different morphology, with much larger domains. Finding the best growth procedure is a very active field of research with technological implications.

\section{2: $C$-face : effect of the rotation between adjacent $C$ layers from ab initio calculations}

As quoted above, all the experimental results go in the same way: on the $\mathrm{C}$ face of $\mathrm{SiC}$, the many $\mathrm{C}$ layers thick samples present an electronic structure characteristic of an ideal, isolated graphene layer. This can be explained by the peculiar stacking of the $\mathrm{C}$ layers that are rotated on this face and not AB stacked as in graphite. The rotation angles appear to be very sensitive to the growth technique (e. g. in UHV or not) but it is found for both types of samples. Fig.4-a shows the twisted bilayer unit cell for the preferred rotation angles observed in Gatech [31], which is close to $28^{\circ}$ for samples prepared by a furnace growth technique (at $1430^{\circ} \mathrm{C}$ in secondary vacuum [5]). We have calculated its band structure using VASP (Fig.4(b), black line). It markedly differs from an AB-bilayer band structure (blue dashed line). Indeed, a twisted bilayer presents a linear dispersion identical to an isolated graphene one (red dots) in the vicinity of the $\mathrm{K}$ point. The rotation electronically decouples the graphene layers, a result which is found for either small or large twist angles [29-32]. 
Figure 4 : Effect of a twisted stacking on the band structure of a graphene bilayer. (a) twisted bilayer unit cell. The chosen angle corresponds to the one observed in furnace grown samples in Gatech, Atlanta. (b) corresponding band structure : the twisted bilayer (black full line) presents a linear dispersion identical to the free graphene one (red dotted line) in the vicinity of the $\mathrm{K}$ point. The dispersion of a bilayer with $A B$ stacking is given for comparison (blue dashed line).
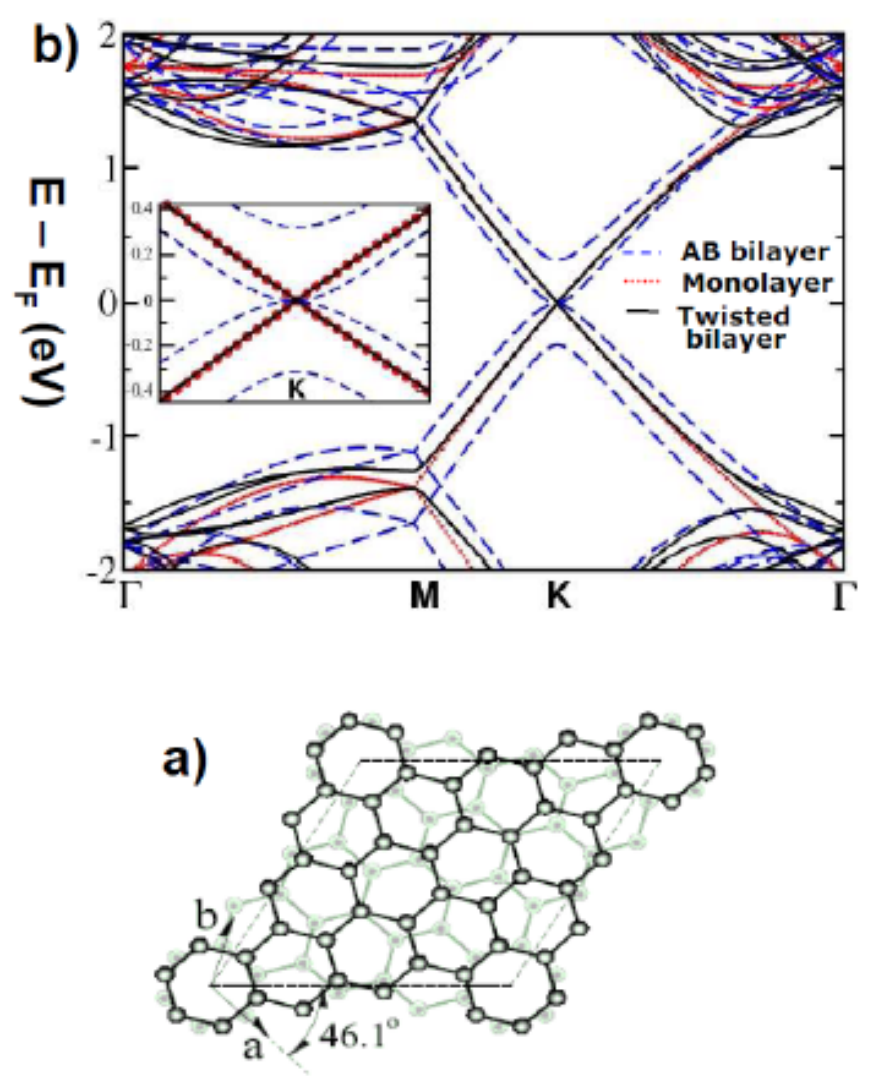

\section{3: Experimental evidence for the electronic decoupling}

Magneto-absorption [24, 33-36] and Raman scattering studies [25] provide the experimental evidences that the electronic structure of few layer graphene on $\mathrm{C}$ terminated surface of $\mathrm{SiC}$ (FLG-C-SiC) resembles more the one of a single graphene sheet than the Bernal-stacked (AB) graphite layers. Faugeras et al. [25] have reported results of micro-Raman scattering studies of FLG-C-SiC samples, and compared them with the data obtained on disks made of highly oriented pyrolitic graphite (HOPG) with different thicknesses and with the spectra of an exfoliated graphene monolayer on $\mathrm{Si} / \mathrm{SiO}_{2}$ substrate. Two most pronounced Raman scattering features which are characteristic of any two-dimensional allotropes of carbon are commonly denoted as G- and 2D-bands. The $\mathrm{G}$ band at $\sim 1582 \mathrm{~cm}^{-1}$ is due to first order (one phonon) Raman scattering process. 
Figure 5 : Characteristic forms of 2D-band observed in Raman scattering on FLG-C-SiC, as compared to the shape of this band seen for graphite and exfoliated graphene.

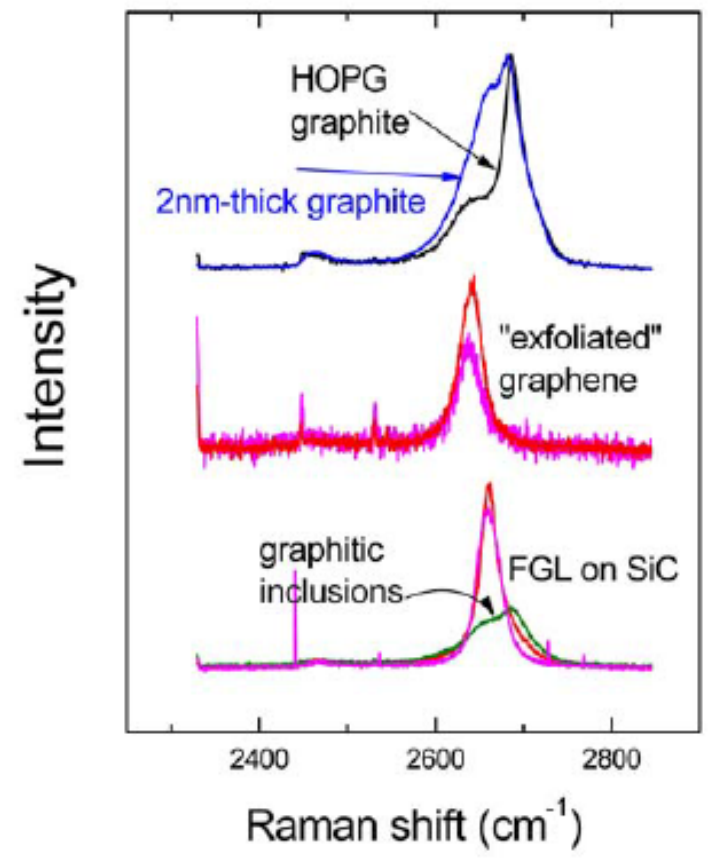

It is characteristic of $\mathrm{sp}^{2}$ hybridization and involves the in-plane optical phonon $\mathrm{E}_{2 \mathrm{~g}}$ near the $\Gamma$ point of the phonon band structure. The 2D-band (at $\sim 2650 \mathrm{~cm}^{-1}$, under HeNe laser excitation) results from a double resonance Raman scattering process, which involves two zone boundary phonons (at $\sim 1325 \mathrm{~cm}^{-1}$ ). This latter Raman scattering process is inherently sensitive to the multi- or single-band character of the dispersion relations of the electronic states. In consequence, the $2 \mathrm{D}$-feature is known to be composed of at least two-components in graphite and also in other graphene multi-layers with Bernal (AB) stacking due to the multi-band character of the electronic states in these systems. In contrast, the unique single Lorentzian form of the 2D-feature is the signature of a system with a single-band electronic dispersion, such as the representative graphene monolayer. Experiments have shown that apart from some residual inclusions, most of the surface of the FLG-C-SiC probed with micro-Raman spectroscopy displays a single component $2 \mathrm{D}$ feature, as for an exfoliated graphene monolayer but in striking contrast to the doublecomponent 2D-feature observed in HOPG disks (see Figure 5). This observation supports the view that FLG-C-SiC is a system which displays single band (Dirac-like) dispersion relation of electronic states characteristic of electronically decoupled graphene layers. It is worth noticing that the energy of the single component 2D-feature observed for FLG$\mathrm{C}-\mathrm{SiC}$ is somewhat higher than the one found for the single graphene sheet. This is consistent with systematic observations of slightly smaller Fermi velocity of Dirac fermions in FLG-C-SiC as compared to the case of graphene. 
Figure 6: Schematic view of Dirac-like dispersion relations (left panel) of two-dimensional electronic states and the resulting Landau level structure (right panel). Optically active inter Landau transitions are marked with vertical arrows.

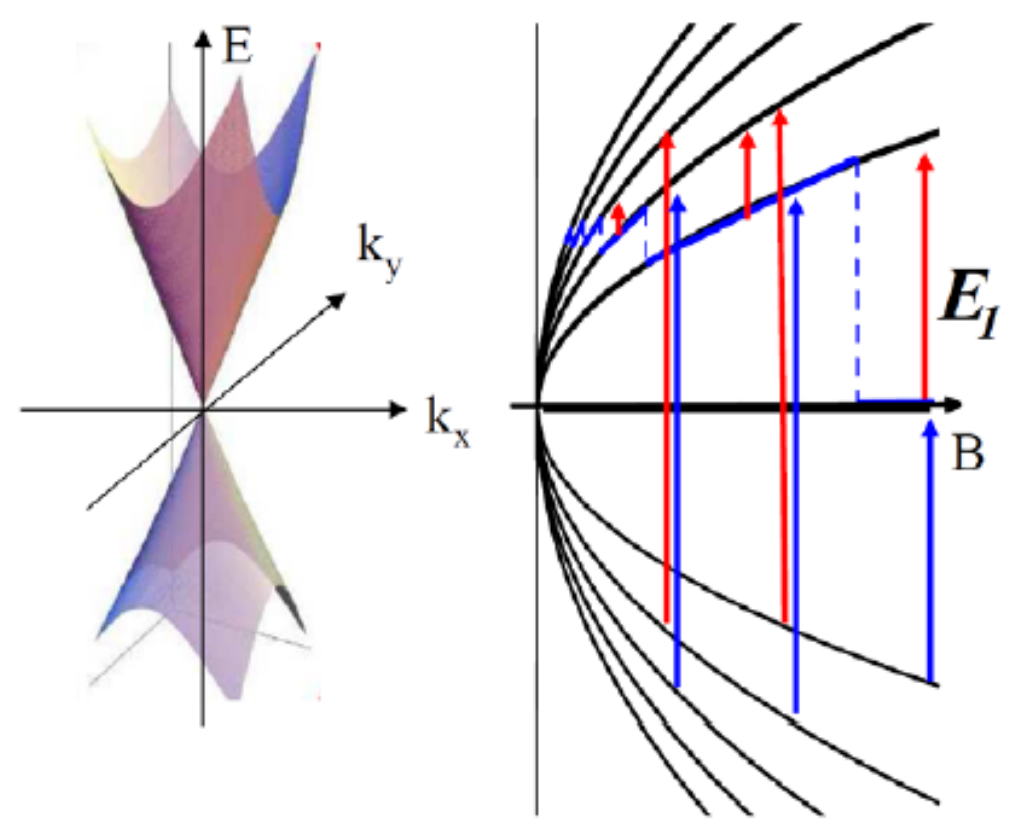

The dependence of the energy $E$ of electrons on their momentum $\vec{p}$ (dispersion relations) can be effectively probed with magneto-spectroscopy methods (see Figure 6). The dispersion relation of two-dimensional electrons of the form of the Dirac cone:, $E(\vec{p})= \pm \widetilde{c}|\vec{p}|$, where $\widetilde{c}$ is the effective (Fermi) velocity, translates into a characteristic sequence of discrete Landau levels $L_{n}$ of energy $E_{n}$ when the magnetic field $B$ is applied in the direction across the layers: $E_{n}=\operatorname{sgn}(n) \widetilde{c} \sqrt{2 e \hbar B|n|}=\operatorname{sgn}(n) E_{1} \sqrt{|n|}$, where $n$ scans all positive and negative integers and-very importantly-zero. $E_{1}$ may be understood as a characteristic energy introduced by the magnetic field. The square root dependence on $\mathrm{B}$ and Landau level index $\mathrm{n}$, a notable fingerprint of dispersion relations of graphene, has been uncovered in FLG-C-SiC with the investigations of inter-Landau transitions, reported by Sadowski et al, [24] as well as transport measurements.

FLG-C-SiC may consist of many, up to $\sim 100$ layers. It is highly conducting in the very close vicinity of the interface with $\mathrm{SiC}$, as a result of charge transfer from $\mathrm{SiC}$. The charge density in subsequent layers decreases rapidly. Electrical measurements on FLG$\mathrm{C}$-SiC samples probe mostly the low resistance interface layers. On the other hand, the quasi-neutral part of the FLG-C-SiC is investigated with optical absorption techniques in an applied magnetic field.

A typical magneto-transmission of FLG-C-SiC consists of several absorption lines which are observed in a wide -from far infrared to nearly visible- spectral range and in a wide range of magnetic fields. 
Figure 7: Magnetic field evolution of energetic centres of the magneto-absorption lines observed for few graphene layers on $\mathrm{C}$-terminated surface of $\mathrm{SiC}$. Note the characteristic sequence and almost perfect, linear with square root of magnetic field dependence -an experimental proof for electronic decoupling of layers.

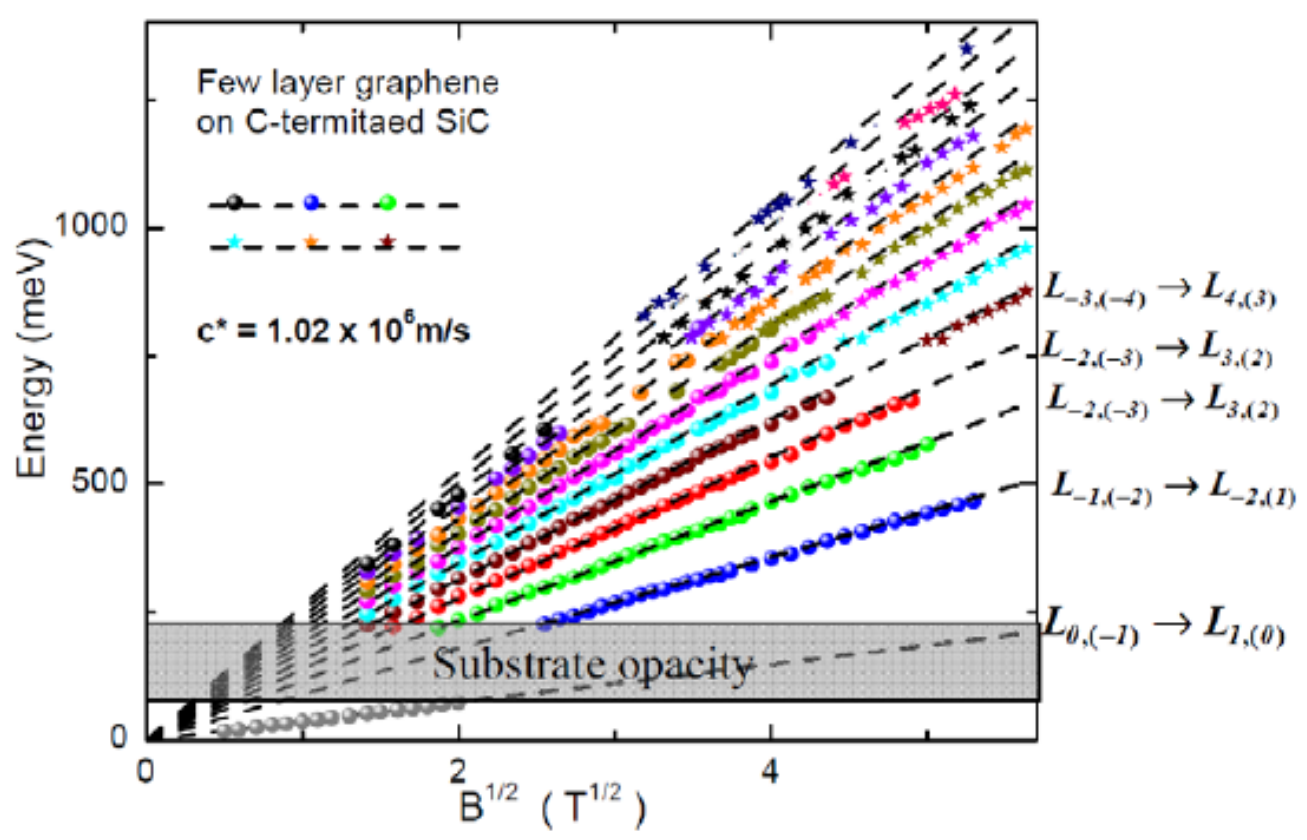

As shown in Figure 7, these lines are centred at energies which in the first approximation follow the characteristic field dependence: linear with the square root of the magnetic field. The observed magneto-absorption lines can be easily identified with optically active: $L_{0,(-1)} \rightarrow L_{1,(0)}, L_{-1,(-2)} \rightarrow L_{2,(1)}, L_{-2,(-3)} \rightarrow L_{3,(2)}, L_{-3,(-4)} \rightarrow L_{2,(3)}, \ldots$, inter Landau level transitions. The image of a simple, Dirac-like dispersion relations, characteristic of a single graphene sheet, applies perfectly well also in case of FLG-C$\mathrm{SiC}$. This again points out the electronic decoupling between the sheets of FLG-C-SiC. Using the linear upon square root of B approximation, the description of the full fan-chart of inter Landau level transitions observed for FLG-C-SiC samples implies a single adjustable parameter, namely the effective (Fermi) velocity $\tilde{\boldsymbol{c}}$. This velocity is found to be slightly sample dependent but remains in the range $\tilde{c}=1.02( \pm 0.02) \cdot 10^{6} \mathrm{~m} / \mathrm{s}$, and is systematically smaller than the corresponding values reported for graphene exfoliated on $\mathrm{Si} / \mathrm{SiO}_{2}$ substrates. Differences in the magneto-optical response of in FLG-C-SiC and of Bernal-stacked graphite layers have been specified in the work of Orlita et al. [34].

Plochocka et al. [35] have shown that Landau level transitions in FLG-C-SiC persist up to nearly visible spectral range. Only far apart from the Dirac point, at energies bigger then $\pm 0.3 \mathrm{eV}$, a clear departure from the linear character of the dispersion relations is observed. This has been shown to be well accounted by the effects of trigonal warping and higher order band corrections.

In a recent work, Orlita et al. [36] have studied the far-infrared magnetoabsorption of FLG-C-SiC in the limit of low magnetic field and high temperature. Focusing on carrier scattering processes, the majority graphene layers of FLG-C-SiC with 
a low carrier concentration have been shown to display an extraordinary quality, expressed by the temperature independent (up too 300K) carrier mobility in excess of $250,000 \mathrm{~cm}^{2} /(\mathrm{V} . \mathrm{s})$. FLG-C-SiC was concluded to be an interesting material to probe the properties of Dirac particles in the immediate vicinity of Dirac point. Record room temperature mobilities give good prognostics for high performance graphene-based electronics.

\section{Transport properties of epitaxial graphene on the $\mathrm{C}$-face}

The epitaxial graphene can be patterned using conventional lithography and plasma etching. Different shape of structures can be obtained $[3,22]$. We have realised ac electrical measurements down to $50 \mathrm{mK}$ in the mixing chamber of a dilution refrigerator under magnetic field applied perpendicular to the sample axis. Here, we present transport measurements realised in hall bar geometry (figure 8).

Figure 8: Hall bar structure etched in epitaxial graphene. a) Typical picture obtained by scanning electron microscope. Using the different contact pads, the longitudinal and the hall resistance can be measured. B) Configuration of the contact for the measurements of the Shubnikov-de-Haas oscillations measurements. c) Configuration of the contacts for the Universal Conductance Fluctuations measurements.

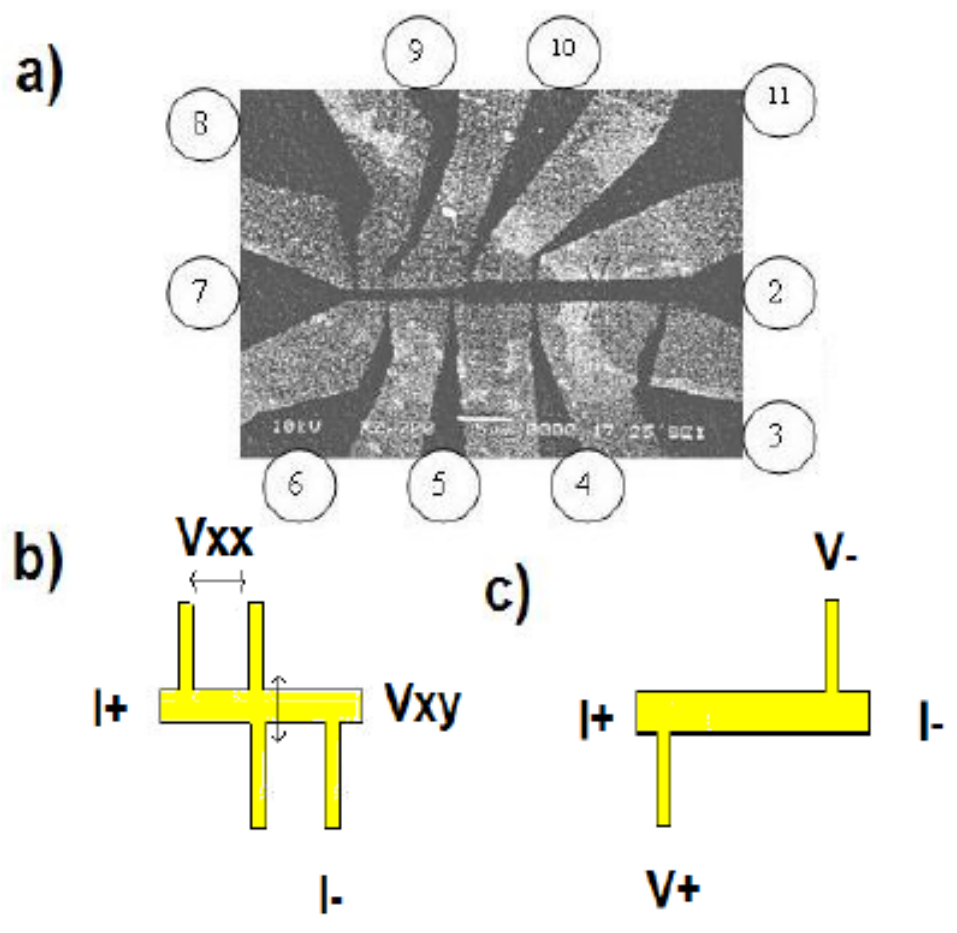

This section is organized as follows: in a first step, the magnetoresistance oscillations are analysed in term of Landau level quantization in order to demonstrate the carriers in epitaxial graphene are Dirac fermions. Then we focus on the fine structure of the magneto conductance which is related to quantum interferences. 
At sufficiently low temperatures and high magnetic fields, the $2 \mathrm{D}$-electrons have a discrete energy spectrum $E_{n}$ (the Landau Level spectrum) due to the quantization of the cyclotrons orbits. In graphene: $E_{n}(B)=\sqrt{ }\left(2 n e B v_{F}^{2} \hbar\right)$. When the magnetic field strength is increased, the split Landau levels move to higher energy. As each energy level passes through the Fermi energy, it depopulates yielding oscillation of the DOS. This causes the material's transport properties to oscillate periodically in $1 / \mathrm{B}$, producing a measurable oscillation in the longitudinal resistivity -the Shubnikov-de-Haas oscillations- and plateaus in the Hall resistivity in large magnetic fields. For the longitudinal and the Hall resistance measurement, a small ac-current is applied through the sample and the transverse and longitudinal voltages are measured using a lock-in amplifier. The magnetoresistance and the Hall resistance are then obtained as the ratios $R_{x x}=V_{x x} / I$ and $R_{x y}=V_{x y} / I$, respectively. From the geometrical shape of the hall bar, we deduce the sheet resistivity $\mathrm{r}_{\mathrm{xx}}=\mathrm{R}_{\mathrm{xx}}{ }^{*} \mathrm{~L} / \mathrm{W}$.

The dependence of the sheet resistance $r_{x x}$ and $r_{x y}$ on magnetic field at $150 \mathrm{mK}$ is presented in Figure 9. The Shubnikov de Haas oscillation increase progressively and are $30 \%$ of the total resistivity at the highest field. The maxima of these oscillations are observed at magnetic field $\mathrm{B}_{\mathrm{n}}$ such as $E_{n}\left(B_{n}\right)=\mathrm{E}_{\mathrm{F}}$. Each maxima is labeled with a landau index $n$ and plotted as a function of $1 / B_{n}$ in a Landau plot of the Figure 10. Due to the Landau quantization, $B_{n}=B_{0} / n$, where $B_{0}=E_{F}^{2} /\left(2 e v_{0}^{2} \hbar^{2}\right)=k_{F}^{2} / 2 e$. The peaks in the Landau plot define a straight line : $B_{n}{ }^{-1}=(n+g) B_{0}^{-1}$. All the characteristic values of the transport in epitaxial graphene are summarized in Table 1.

Table 1: Characteristic value of the Fermi wave vector, Fermi energy, density of carriers and mobility.

\begin{tabular}{|l|l|l|l|l|l|}
\hline $\mathrm{B}_{0}$ & $\mathrm{k}_{\mathrm{F}}$ & $\lambda_{\mathrm{F}}$ & $\mathrm{E}_{\mathrm{F}}$ & $\mathrm{n}_{\mathrm{s}}$ & $\mu$ \\
\hline $\begin{array}{l}\text { Shubnikov de } \\
\text { Haas period }\end{array}$ & $\begin{array}{l}\text { Fermi wave } \\
\text { vector }\end{array}$ & $\mathrm{hv}_{\mathrm{F}} / \mathrm{E}_{\mathrm{F}}$ & $\begin{array}{l}\text { Fermi } \\
\text { energy }\end{array}$ & density & mobility \\
\hline $0.026 \mathrm{~T}^{-1}(38.5 \mathrm{~T})$ & $3.41 \times 10^{8} \mathrm{~m}^{-1}$ & $18.4 \mathrm{~nm}$ & $225 \mathrm{meV}$ & $3.67^{*} 10^{16} \mathrm{~m}^{-2}$ & $0.89 \mathrm{~m}^{2} \mathrm{~V}^{-1} \mathrm{~s}^{-1}$ \\
\hline
\end{tabular}

From the slope we define $k_{F}$ and $n_{\mathrm{s}}=4 k_{F}^{2} / 4 \pi=3.67 \times 10^{12}$ carriers $/ \mathrm{cm}^{2}$. This carrier density is consistent with doped graphene $[3,22,23]$. At this density, the electrons Fermi wavelength is $\lambda_{\mathrm{F}}=18.4 \mathrm{~nm}\left(\mathrm{E}_{\mathrm{F}}=225 \mathrm{meV}\right)$ and the mobility $\mu=0.89 \mathrm{~V}^{2} \mathrm{~m}^{-1} \mathrm{~s}^{-1}$. From the intercept of the straight line at zero, we find $g=0.049$, close to zero, the value expected for Dirac fermions. It is not consistent with $g= \pm 0.5$, the value for normal electrons. This $g$ value demonstrate that the epitaxial grapheme has Dirac crossing which has been also observed in exfoliated single graphene layers [37;38]. This can be considering as surprising since the epitaxial graphene studied consist of several stacked graphene planes. An electron doped plane at the $\mathrm{SiC} /$ graphene interface carrying the main part of the current. Since the screening length is of 1-2 interlayer spacing, conduction is dominated by the interface planes. The planes are rotationally stacked and the relative rotation strongly diminishes the effective coupling between the planes as compared to the case of AB (Bernal) stacking [31]. On the other hand, the Hall resistance shows some steps features but not well defined plateaus. To elucidate this central question, we are currently studying the Hall effect in a contacless Corbino geometry. 
Figure 9: Top panel : Longitudinal -black curve) and Hall resistivity (red curve) measured at 180 $\mathrm{mK}$. Bottom panel : $\rho_{\mathrm{XX}}$ with a smooth background subtracted. The magneto-resistance peaks are showed using arrow.

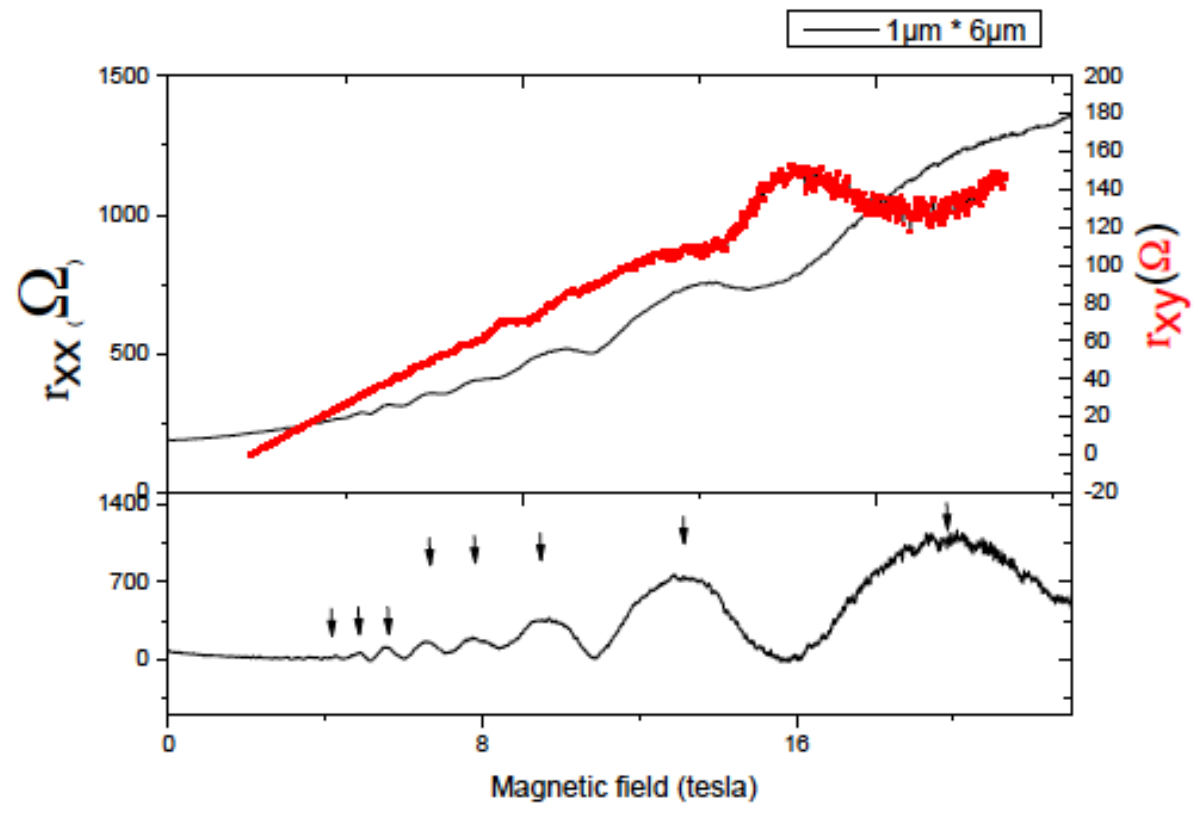

Figure 10: Landau plot. For each maximum of the longitudinal resistance the value of / $\mathrm{Bmax}$ is plotted as a function of the Landau index.

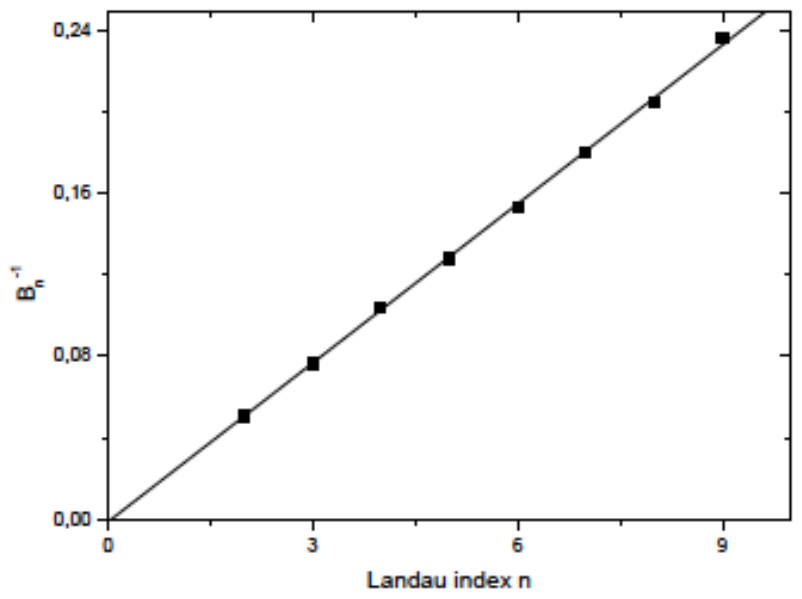


A possible interpretation is proposed in [39] where the scattering of electrons between the doped and undoped planes induces a scattering rate that depends linearly on the magnetic field.

Figure 11: Magneto-fingerprints measured at $50 \mathrm{mK}$. Blue and red curves are magnetoconductance fluctuations for the interchanged voltage and current probes.

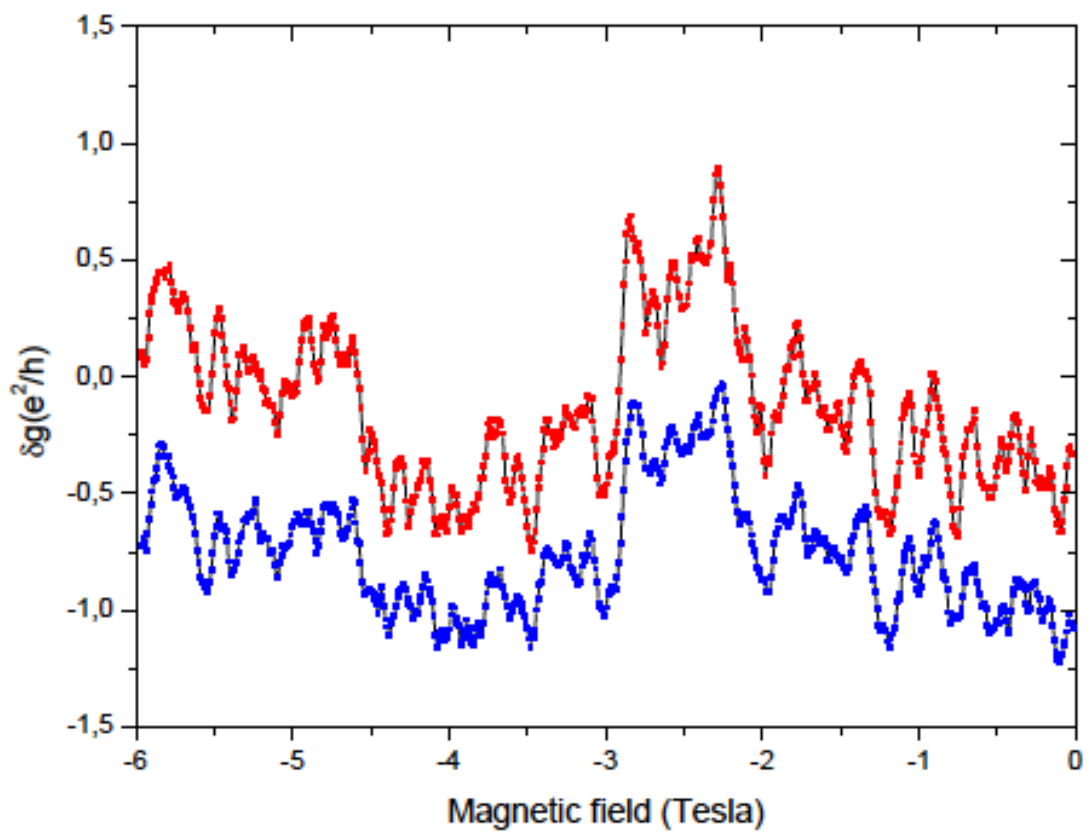

We now analyse the magnetoconductance at $50 \mathrm{mK}$ of a second sample using the four probes geometry shown in Figure 8.c. This sample has an especially low resistance of $100 \mathrm{ohm}$ at low temperature. The origin of such exceptional behaviour is uncertain but we surmise the graphene layers are slightly lifted from the interface thus reducing the scattering. The two magnetoresistance traces for upward and downward magnetic field sweeps are reproducible down to the finest features. The Fourier power spectrum of these fluctuations confirms their aperiodicity.

Theses reproducible and aperiodic fluctuations are the fingerprints under magnetic field of the microscopic realisation of the disorder in the sample. These Universal Conductance Fluctuations (UCF) are related to the interference between all the coherent trajectories inside the sample. A characteristic property of UCF are their symmetries under time-reversal : when $B$ changes to $-\mathrm{B}$ and voltage and current probes are

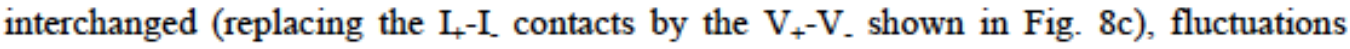
should be identical. As shown in Fig. 11, the time-reversed magneto-conductance traces accurately overlap. The UCF have been recorded at temperatures ranging form $50 \mathrm{mK}$ to $8 \mathrm{~K}$. They can be characterized by their average amplitudes $\pm \delta g_{\mathrm{mms}}$ and the characteristic field $B_{\text {corr }}$ of their auto-correlation function. 
Figure 12: Autocorrelation function of the UCF. The characteristic field is significantly larger than the geometric field $\phi_{0} / \mathrm{wL}$.

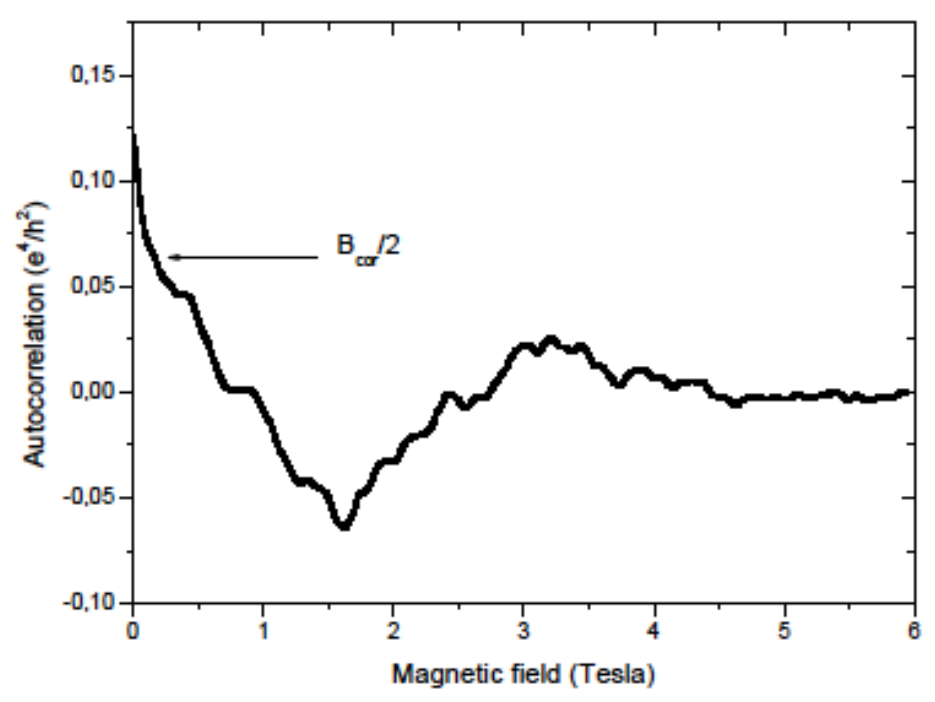

Figure 13 Temperature dependence of the amplitude of the UCF. The amplitude saturates at $0.5 \times \mathrm{e}^{2} / \mathrm{h}$ below $2 \mathrm{~K}$

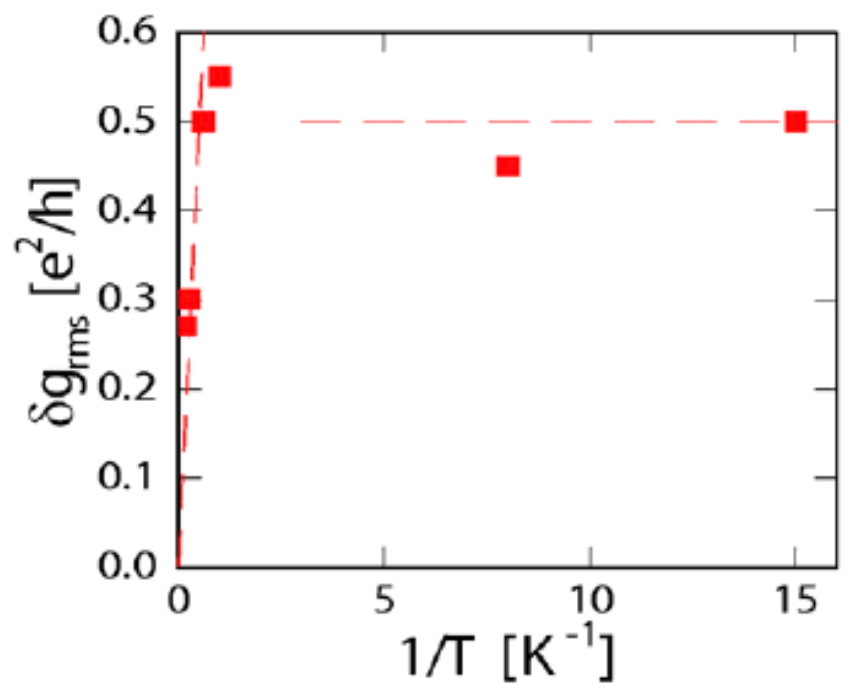

In diffusive conductors, conductance fluctuations have a universal amplitude of $e^{2} / h$ and the width of their auto-correlation, $B_{\text {cor }}=\phi_{0} / w L$ ( $1.7 \mathrm{mT}$ for our sample), is set by the flux quantum through the sample. As shown on the inset of Fig. 11, the rms amplitude $\pm \delta g_{\text {m }}$ saturates at low temperature to a value of $0.5 e^{2} / h$ while the width of the autocorrelation function at $50 \mathrm{mK}$ is $B_{\text {cor }}=0.18 \mathrm{~T}$ (Fig. 12). 
These numbers, not really consistent with a diffusive motion, are now shown to be close what is expected if the electron dynamics through the sample is quasi-ballistic. The amplitude of conductance fluctuations in ballistic cavities have been computed [41] and are significantly smaller than in diffusive conductor, namely $0.25 \times e^{2} / h$ for orthogonal ensembles (small fields) and $0.125 \times e^{2} / h$ for unitary ensembles (large fields). Experiments [42] on a GaAs lithographically defined stadium find an amplitude $0.16 e^{2} / h$ consistent with this theory. For graphene, these numbers should be doubled because of the valley degeneracy. In the experiment the measured amplitude is close to $0.5 e^{2} / h$ and therefore consistent with a chaotic dynamics in this narrow wire.

We now discuss the temperature dependence of conductance fluctuations. Above $2 K$, the conductance fluctuation amplitude decreases. Two processes contribute to this attenuation. For $L_{\phi}>L$, the sample is fully coherent and conductance fluctuations are not affected, in agreement with Fig. 13 above $\mathrm{T}=2 \mathrm{~K}$. When the coherence length $L_{\phi}$ is smaller than the sample size $\mathrm{L}$, the sample can be broken in $L / L_{\phi}$ independent blocks of size $L_{\phi}$ For a quasi-1D wire, this leads to a decrease in the conductance fluctuations ampitude in $L_{\phi}^{-1 / 2}$. When low frequency electron-electron fluctuations are the dominant source of decoherence, the temperature dependence of the phase coherence length is in the diffusive regime $L_{\phi}=(L \hbar D / k T)^{1 / 3}$, leading to a very slow decrease $\left(\mathrm{T}^{-1 / 6}\right)$ of the conductance fluctuation amplitudes[43]. In the ballistic regime, the temperature dependence of $L_{\phi}$ has only been investigated for quasi-1D Luttinger wires where $L_{\phi}=h v_{F} / \eta \pi k T$ decreases only linearly with temperature[44] ( $\eta$ is a dimensionless parameter dependent on the interaction strength). Although the temperature dependence of the UCF has only been measured between 2 and $10 \mathrm{~K}$, this dependence appears to be most consistent with the observed dependence (Fig. 13).

The magneto-transport in a Hall bar epitaxial graphene shows Shubnikov-de-Haas oscillations which are consistent with doped graphene. In agreement with previous observation in epitaxial graphene at smaller magnetic fields (0-9 T), the Landau plot is consistent with a Berry's phase of $\pi$ and permits to conclude that the carriers in epitaxial graphene are Dirac fermions.

The universal conductance fluctuations are an experimental signature of the large phase coherence in this new system. Their characteristic (amplitude and autocorrelation function) give an evidence for the quasi ballistic nature of the electronic transport at least in this very high mobility sample.

\section{Conclusions}

Epitaxial graphene is a new material with considerable potential for nano-electronics. It can be grown over large areas and major improvement in the material quality have already been achieved over a short time. Already, this material is competitive with semiconductor MOSFETs which mobilities (below 40000) have always been limited by the insertion of dopants.

Epitaxial graphene consist of several weakly coupled layers. The experiments reviewed here show that this multilayer structure does not affect the main characteristics of graphene (linear dispersion close to $\mathrm{K}$ and $\mathrm{K}$ ' points) and their consequences (Landau 
level structure, antilocalization etc...). On the other hand, some questions relative to the incomplete Hall effect are open and the specific structure of this material may be important. Furthermore, the presence of partially charged or neutral are bound to have some physical consequence and may lead to the formation of new many-body electronic states. This is a very exciting prospect for the physics of two-dimensional electronic systems.

There are also many developments to come in the area of device physics, going from graphene transistors, quantum dots, hybrid structures, etc. Many of them are underway in Grenoble and Rhône-Alpes will play a leading part in fundamental studies as well as applications of epitaxial graphene.

\section{References}

1 Van Bommel, A.J., Crombeen, J.E. and Van Tooren, A., (1975) 'LEED and Auger electron observations of the $\mathrm{SiC}(0001)$ surface', Surf. Sci., Vol. 48, No 2, pp. 463-472.

2 Forbeaux, I., Themlin, J.-M. and Debever, J.-M., (1999) 'High-temperature graphitization of the $6 \mathrm{H}-\mathrm{SiC}(000-1)$ face', Surf. Sci., Vol. 442, No 1, pp. 9-18.

3 Berger, C., Song, Z., Li, T., Li, X., Ogbazghi, A. Y., Feng, R., Dai, Z., Marchenkov, A. N., Conrad, E. H., First, P. N. and de Heer, W. A., (2004) 'Ultrathin epitaxial graphite: 2D electron gas properties and a route toward graphene-based nanoelectronics', J. Phys. Chem. B, Vol. 108, No 52, pp. 19912-19916.

4 Hass, J., de Heer, W. A. and Conrad, E. H., (2008) 'The growth and morphology of epitaxial multilayer graphene', J. Phys.: Condens. Matter., Vol. 20, No 32, pp. 323202 1-27.

5 Hass, J., Feng, R., Li, T., Zong, Z., de Heer, W. A., First, P. N., Conrad, E. H., Jeffrey, C. A. and Berger, C., (2006) 'Highly ordered graphene for two dimensional electronics', Appl. Phys. Lett., Vol. 89, pp. 143106 1-3.

6 Tsai, M. H., Chang, C. S., Dow, J. D. and Tsong, I. S. T., (1992) 'Electronic contribution to scanning tunneling microscopy images of an annealed $\beta-\mathrm{SiC}(111)$ surface', Phys. Rev. B, Vol. 45,No 3, pp. 1327-1332.

7 Kresse, G. and Hafner, J., (1993) 'Ab initio molecular dynamics for liquid metals', Phys. Rev. $B$, Vol. 47 , No 1 , pp. $558-561$.

8 Varchon F., Mallet P., Veuillen J.-Y, Magaud L. (2008), 'Ripples in epitaxial graphene on the Si-terminated SiC (0001) surface', Phys. Rev. B Vol 77, No 23, pp.235412 1-8.

9 Varchon F., Feng R. Hass J. , Li X., Ngoc Nguyen B., Naud C., Mallet P., Veuillen J.-Y., Berger C., Conrad E.H., Magaud L., (2007) 'Electronic Structure of epitaxial Graphene Layers on SiC: Effect of the Substrate' Phys. Rev. Lett. Vol 99, No 12, 126805 1-4

10 Zhou, S. Y., Gweon, G.-H., Federov, A., First, P.N., de Heer, W.A., Lee, D.-H., Guinea, F., Castro Neto, A.H. and Lanzara, A., (2007) 'Substrate-induced bandgap opening in epitaxial graphene', Nature Mater., Vol. 6, No 10, pp. 770-775.

11 Bostwick, A., Ohta, T., Seyller, Th., Horn, K. and Rotenberg, E., (2007) 'Quasiparticle dynamics in graphene', Nature Phys., Vol. 3, No 1, pp. 36-40.

12 Emtsev, K.V., Speck, F., Seyller, Th., Ley, L. and Riley, J.D., (2008) 'Interaction, growth, and ordering of epitaxial graphene on $\mathrm{SiC}\{0001\}$ surfaces: A comparative photoelectron spectroscopy study', Phys. Rev. B, Vol. 77, No 15, pp. 155303 1-10.

13 Mallet, P., Varchon, F., Naud, C., Magaud, L., Berger, C. and Veuillen, J-Y., (2007) 'Electron states of mono- and bilayer graphene on $\mathrm{SiC}$ probed by scanning-tunneling microscopy', Phys. Rev. B, Vol. 76, No 4, pp. 041403(R) 1-4. 
14 Rutter, G. M., Guisinger, N. P., Crain, J. N., Jarvis, E. A. A., Stiles, M. D., Li, T., First, P. N. and Stroscio, J. A., (2007) 'Imaging the interface of epitaxial graphene with silicon carbide via scanning tunneling microscopy', Phys. Rev. B, Vol. 76, No 23, pp. 235416 1-6.

15 Hembacher, S., Giessibl, F. J., Mannhart, J. and Quate, C. F., (2003) 'Revealing the hidden atom in graphite by low-temperature atomic force microscopy', Proc. Natl. Acad. Sci. U.S.A., Vol 100, No 22, pp. 12539-12542 and references therein

16 Seyller, T., Emtsev, K. V., Gao, K., Speck, F., Ley, L., Tadish, A., Broekman, L., Riley, J. D., Leckey, R. C. G., Rader, O., Varykhalov, A. and Shikin, A. M., (2006) 'Structural and electronic properties of graphite layers grown on $\mathrm{SiC}(0001)$ ', Surf. Sci., Vol. 600, No 18, pp. 3906-3911.

17 Mizes, H.A. and Foster, J.S., (1989) 'Long-Range Electronic Perturbations Caused by Defects Using Scanning Tunneling Microscopy', Science, Vol. 244, No 4904, pp. 559-562.

18 Ruffieux, P., Melle-Franco, M., Groning, O., Bielmann, M., Zerbetto, F. and Groning, P., (2005) 'Charge-density oscillation on graphite induced by the interference of electron waves', Phys. Rev. B, Vol. 71, No 15, pp. 153403 1-4.

19 Rutter, G. M., Crain, J. N., Guisinger, N. P., Li, T., First, P. N. and Stroscio, J. A., (2007) 'Scattering and Interference in Epitaxial Graphene', Science, Vol. 317, No 5835, pp. 219-222.

20 Kobayashi, Y. Fukui, K, Enoki, T., Kusakabe, K and Kaburagi, Y., (2005) 'Observation of zigzag and armchair edges of graphite using scanning tunneling microscopy and spectroscopy', Phys. Rev. B, Vol. 71, No 19, pp. $1934061-4$.

21 Brihuega, I., Mallet, P., Bena, C., Bose, S., Michaelis, C., Vitali, L., Varchon, F., Magaud, L., Kern, K. and Veuillen, J.-Y., (2008) 'Quasiparticle Chirality in Epitaxial Graphene Probed at the Nanometer Scale', Phys. Rev. Lett., Vol. 101, No 20, pp. 206802 1-4.

22 Berger, C., Song, Z., Li, X., Wu, X., Brown, N., Naud, C., Mayou, D., Li, T., Hass, J., Marchenkov, A.N., Conrad, E.H., First, P.N. and de Heer, W.A., (2006) 'Electronic confinement and coherence in patterned epitaxial graphene', Science, Vol. 312, no. 5777, pp. 1191-1196.

23 Wu, X., Li, X., Song, Z., Berger, C. and de Heer, W. A., (2007) 'Weak Antilocalization in Epitaxial Graphene: Evidence for Chiral Electrons', Phys. Rev. Lett., Vol. 98, No 13, pp. $1368011-4$.

24 Sadowski, M.L., Martinez, G. , Potemski, M., Berger, C. and de Heer, W.A., (2006) 'Landau Level Spectroscopy of Ultrathin Graphite Layers', Phys. Rev. Lett., vol. 97, no. 26, pp. 266405

25 Faugeras, C. , Nerrière, A., Potemski, M., Mahmood, A., Dujardin, E., Berger, C and de Heer, W.A., (2008) 'Few-layer graphene on $\mathrm{SiC}$, pyrolitic graphite, and graphene: A Raman scattering study', Appl. Phys. Lett., vol. 92, no. 1, pp. 011914

26 Pong, W-T. and Durkan, C., (2005) 'A review and outlook for an anomaly of scanning tunnelling microscopy (STM): superlattices on graphite', J. Phys. D: Appl. Phys., Vol. 38, No 21, pp. R329-R355.

27 Varchon, F., Mallet, P., Magaud, L. and Veuillen, J.-Y., (2008) 'Rotational disorder in fewlayer graphene films on $6 \mathrm{H}$-SiC(000-1): A scanning tunnelling microscopy study', Phys. Rev. B, Vol. 77, No 16, pp. 165415 1-5.

28 Hiebel, F., Varchon, F., Mallet, P., Magaud, L. and Veuillen, J-Y., (2008) 'Graphene-substrate interaction on $6 \mathrm{H}$-SiC(0001): A scanning tunneling microscopy study', Phys. Rev. B, Vol. 78, No 15 , pp. $1534121-4$.

29 Lopes dos Santos, J.M.B., Peres, N.M.R. and Castro Neto, A.H., (2007) 'Graphene Bilayer with a Twist: Electronic Structure', Phys. Rev. Lett., Vol. 99, No 25, pp. 256802 1-4.

30 Shallcross, S., Sharma, S. and Pankratov, O. A., (2008) 'Quantum interference at the twist boundary in graphene', Phys. Rev. Lett., Vol. 101, no. 5, pp. 056803 1-4. 
31 Hass, J., Varchon, F., Millan-Otoya, J.E., Sprinkle, M., Sharma, N., de Heer, W.A., Berger, C., First, P.N., Magaud, L. and Conrad, E.H., (2008) 'Why multilayer graphene on $4 \mathrm{H}$ $\mathrm{SiC}(000 \mathbf{I})$ behaves like a single sheet of graphene', Phys. Rev. Lett., Vol. 100, No 12, pp. $1255041-4$.

32 Latil, S., Meunier, V. and Henrard, L., (2007) 'Massless fermions in multilayer graphitic systems with misoriented layers: $A b$ initio calculations and experimental fingerprints', Phys. Rev. B, Vol. 76, No 20, pp. 201402(R) 1-4.

33 Sadowski, M.L., Martinez, G., Potemski, M., Berger, C. and de Heer , W.A., (2007) 'Magnetospectroscopy of epitaxial few-layer graphene', Solid State Comm., vol. 143, no. 1-2, pp. 123-125.

34 Orlita, M., Faugeras, C., Martinez, G., Maude ,D. K, , Schneider, J. M. , Sprinkle, M., Berger, C. , de Heer, W. A., Potemski, M. (2009) Magneto-transmission of multi-layer epitaxial graphene and bulk graphite: A comparison, Solid State Comm., vol. 149, No. 27-28, pp. 1128

35 Plochocka, P., Faugeras, C., Orlita, M., Sadowski, M.L., Martinez, G., Potemski, M., Goerbig, M.O., Fuchs, J-N., Berger, C., de Heer, W.A. , (2008) 'High-Energy Limit of Massless Dirac Fermions in Multilayer Graphene using Magneto-Optical Transmission Spectroscopy', Phys. Rev. Lett. Vol. 100, No 8 ,pp. 087401 1-4.

36 Orlita, M., Faugeras, C. , Plochocka, P., Neugebauer, P., Martinez, G., Maude ,D. K. , Barra, A-L., Sprinkle, M., Berger, C. , de Heer, W. A., and Potemski, M. (2008) 'Approaching the Dirac Point in High-Mobility Multilayer Epitaxial Graphene', Phys. Rev., Lett. , Vol. 101, No 26 ,pp. 267601 1-4.

37 Novoselov, K. S., Geim, A.K., Morozov, S.V., Jiang, D., Katsnelson, M.I., Grigorieva, I.V., Dubonos, S.V. and Firsov, A.A., (2005) 'Two-dimensional gas of massless Dirac fermions in graphene', Nature, Vol. 438, No 7065, pp. 197-200.

38 Zhang, Y, Tan, Y.-W., Stormer, H.L. and Kim, P., (2005) 'Experimental observation of the quantum Hall effect and Berry's phase in graphene', Nature, Vol. 438, No 7065, pp. 201-2004.

39 Darancet, P. , Wipf, N. , Berger, C. , De Heer, W. and Mayou, D. , (2008) 'Quenching of the Quantum Hall Effect in Multilayered Epitaxial Graphene: The Role of Undoped Planes', Phys. Rev. Lett., Vol. 101, No 11 , pp. 116806 1-4.

40 Baranger, H. U. and Mello, P. A. , (1994) Mesoscopic transport through chaotic cavities: A random S-matrix theory approach, Phys. Rev. Lett., Vol. 73, No 1, pp. 142 -145.

41 Jalabert, R. A. , Baranger ,H. U. and Stone, A. D., (1990) "Conductance fluctuations in the ballistic regime: A probe of quantum chaos?', Phys. Rev. Lett., Vol. 65, No 19, pp. 2442-2245.

42 Marcus, C. M. , Rimberg, A. J. , Westervelt, R. M. Hopkings, P. F. and Gossard, (1992) 'Conductance fluctuations and chaotic scattering in ballistic microstructures', Phys. Rev. Lett., Vol. 69 , No 3, pp. $506-509$.

43 Note that for a $2 \mathrm{D}$ sample in the diffusive regime, e-e interactions also contribute to a decreases of $L_{\phi} \sim T^{1 / 2}$, but now the sample can be broken into $\left(L / L_{\phi}\right)^{2}$ independent blocks leading also to a decrease of the amplitude of the UCF as $\mathrm{T}^{-1 / 2}$.

44 Le Hur, K, (2002) 'Electron fractionalization-induced dephasing in Luttinger liquids', Phys. Rev. B, Vol. 65, No 23, pp. 233314 1-4. 